\title{
Frühpädagogische Ausbildungskonzepte in drei nordischen Ländern
}

\author{
Orientierung für die Weiterentwicklung des \\ Professionsprofils in Deutschland?
}

\section{Pamela Oberhuemer}

Zusammenfassung: Mit der Umsetzung von offiziellen frühpädagogischen Bildungsplänen sowie der gesetzlichen Verankerung eines Platzanspruchs ab 2013 für Kinder im Alter von 1 und 2 Jahren verändern sich die bisherigen Parameter der professionellen Bildungs- und Erziehungspraxis in der frühen Kindheit in Deutschland. Trotz neuerer Ausbildungsinitiativen bleibt das Professionsprofil letztlich ungeklärt. Um diesen Klärungsprozess zu fördern, werden die sich im Umbruch befindenden Hochschulausbildungen und Berufsprofile der Kernfachkräfte in frühpädagogischen Tageseinrichtungen in Dänemark, Finnland und Schweden vorgestellt und mit Bezug zur Situation in Deutschland analysiert. Die Ausbildungen mit der Gemeinsamkeit eines akademischen Abschlusses zielen auf drei unterschiedliche Berufsprofile hin: eine sozialpädagogische Fachkraft außerhalb des Bildungssystems (Dänemark), eine frühpädagogische Fachkraft im Sozialwesen und am Schnittpunkt zum Bildungswesen (Finnland) und eine vor- und grundschulpädagogische Fachkraft innerhalb des Bildungswesens (Schweden). Eine Auseinandersetzung mit diesen unterschiedlichen Profilen könnte zum Klärungsprozess in Deutschland beitragen.

Schlüsselwörter: Ausbildung · Frühpädagogik $\cdot$ Berufsprofil $\cdot$ Nordische Länder

\section{Teacher education for the early years in three Nordic countries: impetus for reconceptualising professional profiles in Germany?}

\begin{abstract}
In Germany, policy drives in recent years to implement first-time offcial early childhood curricula, coupled with new legislation guaranteeing entitlement by 2013 to a place in early childhood provision not only for three to six year olds but also for one and two year olds, are challenging the traditional parameters of professional work in early childhood services. Despite a flurry of initiatives by individual higher education institutions to introduce new forms of professional education/training for key workers, the required and desired professional profile is far from being clear. As one possibility of moving the discussion forward, this paper looks at current study routes and professional profiles of the core practitioners in early childhood centres in Denmark, Finland and Sweden. In each of these three Nordic countries the higher education study route
\end{abstract}

Online publiziert: 16.01 .2010

(C) Die Autoren 2010. Dieser Artikel ist auf Springerlink.com mit Open Access verfügbar.

P. Oberhuemer $(\square)$

Staatsinstitut für Frühpädagogik (IFP)

Winzererstraße 9, 80797 München, Deutschland

E-Mail: Pamela.Oberhuemer@ifp.bayern.de 
leads to a bachelor degree. However. the professional profiles in each country differ considerably, ranging from a social pedagogy professional working outside the education system (Denmark), to an early childhood professional working both within a social welfare and education framework (Finland), or an early childhood and primary school professional operating within the education system (Sweden). Debating these varying profiles could be one way of clarifying both policy goals and the content of new higher education level initiatives in Germany.

Keywords: Early years education/care - Germany $\cdot$ Nordic countries $\cdot$ Professional education/ training Occupational profile

\section{Problemskizze: Die frühpädagogische Ausbildungsdiskussion in Deutschland}

Zwei neuere politische Strategien markieren entscheidende Wendepunkte in der Geschichte der Frühpädagogik in Deutschland: zum einen die sukzessive Verabschiedung von offiziellen Bildungsplänen für den elementarpädagogischen Bereich in allen Bundesländern zwischen 2003 und 2007; zum anderen die gesetzliche Verankerung eines Rechtsanspruchs auf ein Betreuungsangebot für Kinder im Alter von 1 und 2 Jahren ab 2013 (Kinderförderungsgesetz 2009 - KiFöG). Damit verändern sich die Parameter der bisherigen professionellen Bildungs- und Erziehungspraxis deutlich.

Frühkindliche Bildung und Kindertagesbetreuung stehen heute nicht nur im Zentrum des fachwissenschaftlichen und fachpolitischen Interesses einer bisher relativ kleinen Gruppe von frühpädagogischen Expertinnen und Experten, sondern auch im Blickpunkt gesellschaftlicher und politischer Aufmerksamkeit. Als Konsequenz steigen die Erwartungen und Anforderungen an die professionelle Bildungs- und Erziehungsarbeit mit jungen Kindern und auch das Interesse an Qualitätsfragen. Dies ist ein europaweites Phänomen. Als Erkenntnis der Forschung (vgl. Siraj-Blatchford 2004) und auch in der jüngsten OECD-Studie (2006) zur Lage der Kindertagesbetreuung in sechzehn europäischen und vier außereuropäischen Ländern wird das Fachpersonal als „Schlüssel“ zur Bildungs- und Betreuungsqualität hervorgehoben. Dieser qualitative Aspekt fehlte noch bei der Barcelona-Zielvereinbarung der Europäischen Union aus dem Jahre 2002, die sich lediglich mit dem quantitativen Ausbau der Kindertagesbetreuung in den damaligen fünfzehn Mitgliedstaaten befasste. Erst 2007 wurde bei einer Konferenz der europäischen Bildungsministerinnen und Bildungsminister in Heidelberg die qualitative Seite des Ausbaus explizit betont, unter anderem die Ausbildungsqualität des frühpädagogischen Personals (BMBF 2007).

In Deutschland bildet die Berufsgruppe der Erzieherinnen und Erzieher mit $66 \%$ in den westlichen und 90\% in den östlichen deutschen Bundesländern die Kerngruppe des pädagogischen Personals in den Kindergärten, Kinderkrippen und anderen Tageseinrichtungen für Kinder (Autorengruppe Bildungsberichterstattung 2008, S. 55). Fragen der Qualität und Struktur dieser Ausbildung beschäftigen Fachkreise seit über 30 Jahren (vgl. Schrader 1973; Pestalozzi-Fröbel-Verband 1977). Seit dieser Zeit wurden immer wieder Kernaspekte der Ausbildung wie zum Beispiel die Zugangsvoraussetzungen oder die Verschulungstendenzen (vgl. rückblickend: Ebert 2006) oder auch die fehlende Verknüpfung zwischen Ausbildung und Forschung (Oberhuemer 2000, S. 43) kritisiert. Jenseits kurzfristiger Innovationsschübe kam es aber zu keiner nachhaltigen Reformstra- 
tegie. Erst mit der aktuellen Verdichtung von bildungspolitischen und europapolitischen Begründungsmustern ist es zu einer neuen Bewegung in der frühpädagogischen Ausbildungslandschaft gekommen. Seit 2004 entstehen vor allem an den berufsqualifizierenden Hochschulen (Fachhochschulen und Pädagogischen Hochschulen) die ersten frühpädagogischen Studienangebote. In jedem Bundesland gibt es mittlerweile mindestens einen diesbezüglichen Studiengang; inzwischen sind es insgesamt 47 neue Studiengänge mit Bachelor-Abschluss (vgl. WiFF 2009).

Trotz der innovativen Ausbildungsinitiativen der letzten Jahre - wie zum Beispiel der Entwicklung eines curricularen Orientierungsrahmens für Hochschulen durch das PiKProjekt der Robert Bosch Stiftung (2008) - ist insgesamt noch keine klare Linie für die Zukunft erkennbar. Die neuen Studiengänge heißen verschiedentlich: Elementare Pädagogik; Elementarpädagogik; Frühpädagogik; Frühe Bildung; Frühkindliche und Elementarbildung; Bildung und Erziehung von Kindern; Erziehung und Bildung im Kindesalter; Bildung und Erziehung in der Kindheit; Bildung und Förderung in der Kindheit; Management von Erziehungs- und Bildungseinrichtungen; Bildungs- und Sozialmanagement mit Schwerpunkt frühe Kindheit.

Wohin des Weges? Auch ein genauerer Blick auf die europäischen Systeme der professionellen Bildung für eine Tätigkeit im Arbeitsfeld der frühkindlichen Bildung, Betreuung und Erziehung zeigt, dass es aus der Perspektive der Ausbildungspolitik noch sehr unterschiedliche Vorstellungen und Interpretationen von Kernaspekten der Ausbildungsqualität gibt. Diese betreffen unter anderem die formalen Qualifikationsanforderungen für die Arbeit als gruppenverantwortliche frühpädagogische Fachkraft, die Studiengangstrukturen und curricularen Orientierungen der Ausbildung sowie das angestrebte Professionsprofil. Wie das Verständnis dieser Kernaspekte in Dänemark, Finnland und Schweden aussieht, steht im Mittelpunkt dieser länderübergreifenden Analyse. Grundlage ist die SEEPRO-Studie, ein vom Bundesministerium für Familie, Senioren, Frauen und Jugend gefördertes Projekt am Staatsinstitut für Frühpädagogik in München über die Ausbildungen und Arbeitsfelder des Fachpersonals in Kindertageseinrichtungen der Europäischen Union. Diese stützt sich nicht nur auf Sekundärquellen, sondern auch auf beauftragte Expertenbeiträge, die im aktiven Dialog mit Fachwissenschaftlerinnen und -wissenschaftlern in den jeweiligen Ländern entstanden sind (Oberhuemer et al. 2009; Oberhuemer u. Schreyer, im Druck).

Um den breiteren Kontext der frühkindlichen Bildung, Betreuung und Erziehung in Dänemark, Finnland und Schweden und damit auch die Einbettung der professionellen Arbeit in Tageseinrichtungen für Kinder besser verstehen zu können, werden einleitend einige Merkmale der Kita-Systeme in den drei Ländern kurz vorgestellt.

\section{Die Kita-Systeme in Dänemark, Finnland und Schweden}

\subsection{Erwerbstätigkeit von Frauen mit Kindern}

Als Antwort auf die wachsende Erwerbstätigkeit von Müttern mit jungen Kindern begann in Dänemark, Finnland und Schweden, aber auch in der DDR, bereits in den 1970er-Jahren der schrittweise und zügige Ausbau von ganztägigen Tageseinrichtungen für Kinder 
Tab. 1: Müttererwerbstätigkeit in Dänemark, Deutschland, Finnland und Schweden nach Alter der Kinder (Angaben in Prozent)

\begin{tabular}{llll}
\hline & $\begin{array}{l}\text { Erwerbstätigkeit bei } \\
\text { Müttern mit Kindern } \\
\text { zwischen 0 und 2 } \\
\text { Jahren }\end{array}$ & $\begin{array}{l}\text { Erwerbstätigkeit bei } \\
\text { Müttern mit Kindern } \\
\text { zwischen 3 und 5 } \\
\text { Jahren }\end{array}$ & $\begin{array}{l}\text { Erwerbstätigkeit bei } \\
\text { Müttern mit Kindern } \\
\text { zwischen 6 und 11 } \\
\text { Jahren }\end{array}$ \\
\hline EU25 & $\mathbf{5 2}$ & $\mathbf{6 0}$ & $\mathbf{6 7}$ \\
Schweden & 72 & 81 & 76 \\
Dänemark & 71 & 81 & 87 \\
Finnland & 52 & 81 & 85 \\
Deutschland & 31 (Westen) & 54 (Westen) & 65 (Westen) \\
& 41 (Osten) & 64 (Osten) & 64 (Osten) \\
& & & mit Kindern zwischen \\
& & & und 9 Jahren \\
\hline
\end{tabular}

Für Dänemark und Finnland und die EU25-Durchschnittsraten sind die Daten aus dem Jahr 2003 (Eurostat 2005); für Schweden aus dem Jahr 2005 (OECD 2007); für Deutschland aus dem Jahr 2005 (Bundeszentrale für politische Bildung 2008)

in den Jahren vor der Pflichteinschulung. In den heutigen westdeutschen Bundesländern trat der politische Wille zum konsequenten Ausbau erst über zwanzig Jahre später ein.

Heute liegt in allen drei nordischen Ländern die Erwerbstätigkeitsrate für Mütter mit Kindern bis zum vollendeten 12. Lebensjahr weit über dem EU-Durchschnitt. In Deutschland dagegen liegt die Quote insbesondere bei Müttern mit Kindern bis zu 3 Jahren deutlich unter dem EU-Durchschnitt (vgl. Tab. 1).

Die im Vergleich zu Dänemark und Schweden niedrige Beteiligung der Mütter mit Kindern zwischen 0 und 2 Jahren in Finnland (die trotzdem dem EU-Durchschnitt entspricht) ist vielleicht durch zwei Phänomene zu erklären: Zum einen gibt es in Finnland nur wenig Teilzeitstellen, während 2005 in Schweden 50\% der Mütter mit einem Kind unter 2 Jahren teilzeitbeschäftigt waren (Haas et al. 2008); zum anderen können finnische Mütter den seit 1996 geltenden staatlichen Betreuungszuschuss für die Betreuung eines unter 3-jährigen Kindes zu Hause in Anspruch nehmen (Hännikäinen 2009).

\subsection{Administration und Organisation der Kindertagesbetreuung}

In allen drei nordischen Ländern überwiegen öffentliche, im geringeren Ausmaß auch öffentlich geförderte Tageseinrichtungen in privater Trägerschaft und es existieren kaum privat-gewinnorientierte Einrichtungen; in allen bildet die Tagespflege einen integralen Teil des öffentlichen Systems der frühkindlichen Bildung, Betreuung und Erziehung; und in allen bezahlen die Eltern einen Teil der Kosten für die Dienstleistung, wobei dieser Anteil vor allem in Schweden, aber auch in Dänemark zunehmend reduziert wird.

In Dänemark ist das System der Kindertagesbetreuung (dagtilbud) Teil des sozialen Wohlfahrtssystems. Für die Kindertageseinrichtungen (Kinderkrippen, Kindergärten, altersübergreifende Tageseinrichtungen) und für die Kindertagespflege (Tagespflegestellen und Tagespflegenetzwerke) ist die aktuelle zuständige nationale Behörde das Innen- und Sozialministerium. Die Kommunen sind für die örtliche Administration und Organisation 
der frühkindlichen Bildung, Erziehung und Betreuung verantwortlich und dazu verpflichtet, einen Platz für alle Kinder zwischen 6 Monaten und 6 Jahren sicher zu stellen.

Ähnlich organisierte Strukturen für diese Altersgruppe sind auch in Finnland und Schweden zu finden, allerdings mit unterschiedlichen Akzenten. Während in Finnland die Kindertagesbetreuung wie in Dänemark Teil des Wohlfahrtssystems ist, wurde 1996 in Schweden die Entscheidung gefällt, die Zuständigkeit vom Ministerium für Gesundheit und Soziales zum nationalen Bildungsministerium zu transferieren (Allodi 2007, S. 1). Die Nationale Bildungsagentur (Skolverket) ist nun nicht nur für die Fachaufsicht und Weiterentwicklung im Schulbereich, sondern auch im Kita-Sektor zuständig. Diese Entscheidung war eine Konsequenz der allgemeinen Bildungsdebatte der 1990er-Jahre und hatte deutliche Auswirkungen auf eine Reform der Ausbildung für frühpädagogische Fachkräfte. Im Jahr 2005 folgte Norwegen als zweites nordisches Land dem schwedischen Beispiel. Auch in Finnland ist ein Trend zum Ressortwechsel zu beobachten. Während auf nationaler Ebene das Sozialministerium noch Verantwortung für den frühkindlichen Bereich trägt, wird in den Kommunen im Rahmen von verwaltungstechnischen Umstrukturierungen die Verantwortung für die Kindertagesbetreuung zunehmend an den Bildungsbereich übergeben. Bis Oktober 2008 hatten 20\% der Kommunen diesen Schritt vollzogen (Onnismaa 2009).

Die Bildungsbehörden sind auch für die in allen drei Ländern vorfindbaren und bisher nichtpflichtigen Übergangsklassen für 6-Jährige, in denen die Kita-Kernfachkräfte gleichfalls arbeiten, verantwortlich. In Dänemark wurden bereits in den 1980er-Jahren die ersten Vorschulklassen für 6-Jährige an Grundschulen eingeführt, die - im Gegensatz zu den Tageseinrichtungen für jüngere Kinder - kostenfrei waren. Schweden folgte diesem Beispiel einige Jahre später, Finnland im Jahre 2001. In Finnland werden diese Übergangsklassen entweder in der Tageseinrichtung oder in der Schule angesiedelt: die große Mehrheit (79\%) befindet sich in Tageseinrichtungen. In Dänemark wurde mit dem Schuljahr 2008/09 der Pflichtbesuch für diese Vorschulklassen eingeführt, was einer Herabsetzung der Schulpflicht von 7 auf 6 Jahren gleichkommt.

\subsection{Ganztägige und hohe Partizipationsraten - auch bei den unter 3-Jährigen}

Trotz dieser Unterschiede im Detail überwiegen bei weitem die Ähnlichkeiten zwischen den drei Ländern. In allen besuchen die Kinder überwiegend Ganztageseinrichtungen. In Dänemark lag 2008 die Partizipation der 3- bis 5-Jährigen bei 97\% und der unter 3-Jährigen bei 66\%. 2006 wurde knapp die Hälfte der 1- bis 2-Jährigen in der Kindertagespflege betreut, während fast alle 3- bis 5-Jährige eine Tageseinrichtung besuchten. Im gleichen Jahr wurden $66 \%$ der Kinder unter 3 Jahren ganztags für mehr als 30 Stunden wöchentlich betreut und weitere 7\% bis zu 29 Stunden. In der Altersgruppe der 3jährigen Kinder bis zum Schuleintritt waren dies 80\% bzw. 16\% (Eurostat 2008; Statistics Denmark 2009). In Finnland liegen die Partizipationsraten niedriger. Nach dem OECD-Länderprofil Finnland (OECD 2006) besuchen 27,5\% der 1- bis 2-Jährigen, 43,9\% der 2- bis 3-Jährigen, $62,3 \%$ der 3- bis 4-Jährigen, 68,5\% der 4- bis 5-Jährigen und 73\% der 5- bis 6-Jährigen eine Ganztageseinrichtung oder werden in der Kindertagespflege betreut. In Schweden besuchten 2008 knapp über $81 \%$ der 1- bis 5-Jährigen eine frühpädagogische Ganztageseinrichtung (förskola). Während etwa 46\% der 1-Jährigen - im europäischen Vergleich 
ein sehr hoher Anteil - eine Tageseinrichtung besuchen, sind es bei den 2-Jährigen schon über $86 \%$. Da viele Eltern von der kombinierten, bezahlten Elternzeit Gebrauch machen, gibt es in den Tageseinrichtungen sehr wenige Kinder unter 1 Jahr. Auch wenn der Besuch einer Vorschulklasse freiwillig ist, wird sie von ca. 96\% der 6-Jährigen besucht (Karlsson Lohmander 2009).

\subsection{Gruppengröße und Personalschlüssel}

In allen drei Ländern ist die Fachkraft-Kind-Relation im Vergleich zu den meisten anderen europäischen Ländern günstig (Oberhuemer u. Schreyer, im Druck). In Tageseinrichtungen mit unter 3-jährigen Kindern liegt der Personalschlüssel in Dänemark bei 1:3,2 in Gruppen mit 11 bis 12 Kindern, in Finnland bei 1:4 in Gruppen mit 21 Kindern und in Schweden bei 1:5,2 in Gruppen mit 14,6 Kindern. In Tageseinrichtungen mit 3- bis 6-jährigen Kindern liegt der Personalschlüssel in Dänemark bei 1:5,9 in Gruppen mit 20 bis 22 Kindern, in Finnland bei 1:7 ebenfalls in Gruppen mit 20 bis 22 Kindern und in Schweden bei 1:5,2 in Gruppen von 19,7 Kindern. In Schweden werden Gruppengröße und Personalschlüssel von den Kommunen festgelegt; im Unterschied zu Dänemark und Finnland gibt es keine nationalen Standards. Die Einrichtungs- und Gruppenleitungen sind in allen drei Ländern auf Hochschulniveau ausgebildet und die Ergänzungskräfte in Finnland und Schweden auf post-sekundärem oder höherem Sekundarstufenniveau; in Dänemark haben die pädagogischen Assistentinnen keine einschlägige Ausbildung, obwohl eine 25-monatige Qualifikation auf Sekundarstufe II-Niveau möglich ist (Oberhuemer u. Schreyer, im Druck).

\subsection{Einige Ähnlichkeiten und Unterschiede zur Betreuungssituation in Deutschland}

Bei einzelnen Systemmerkmalen sind Ähnlichkeiten zur Situation in Deutschland zu erkennen: die anteilige Kostenübernahme durch Eltern - mit einer Tendenz zur Reduzierung des Elternbeitrags; die gesetzliche Gleichstellung von Kindertageseinrichtungen und Kindertagespflege; der Trend zur früheren Einschulung. Im zentralen Punkt der Investitionen fällt aber der Vergleich weniger günstig aus (vgl. Leu u. Schelle 2009, S. 16). Nach Einschätzungen der OECD (2006, S. 105) über die Ausgaben für vorschulische Bildungs- und Betreuungsangebote für 0- bis 6-Jährige betragen diese für Dänemark 2,1\% des Bruttoinlandsprodukts, für Schweden 1,7\% und für Finnland 1,3\%. Hinzu kommen in Finnland zusätzliche 0,3\% für die Vorschulklassen für 6-Jährige. Nach der gleichen OECD-Einschätzung betragen die Ausgaben in Deutschland etwa 0,45\% des BIP.

Auch der Anteil von Ganztagsplätzen ist in Deutschland vergleichsweise gering: 2006 verbrachten 45,9\% der Kinder unter drei Jahren zwischen 7 und 10 Stunden in einer Tageseinrichtung, bei den Kindern im Alter von drei Jahren bis zum Schuleintritt waren es $24,3 \%$ (Deutsches Jugendinstitut 2008, S. 42 ff.).

Diese kontextuellen Daten sind als Hintergrundinformation für die Einschätzung der Entwicklungen um die frühpädagogischen Berufe wichtig. In Dänemark, Finnland und Schweden wäre es zum Beispiel aufgrund der durchgehend ganztägigen Angebote kaum denkbar gewesen, dass der Beruf der Hauptbeschäftigten in den Tageseinrichtungen sich in erheblichem Ausmaß zu einem Teilzeitberuf entwickelt, wie es zurzeit in Deutschland 
der Fall ist. 2007 waren hierzulande nur 39,7\% des pädagogischen Personals in Kindertageseinrichtungen Vollzeitbeschäftigte (Forschungsverbund DJI/TU Dortmund 2008).

\section{Formale Mindestqualifikation für frühpädagogische Kernfachkräfte}

Während in Deutschland die Erzieherinnen als Kernfachkräfte, d.h. in der Funktion als Gruppen- oder Einrichtungsleitung, auf post-sekundärem Niveau an berufsbildenden Fachschulen/Fachakademien ausgebildet werden, studieren ihre Kolleginnen in Dänemark, Finnland und Schweden an Hochschulen bzw. Universitäten. Dänemark hat mit 60\% die höchste Quote von akademisch ausgebildetem Kita-Personal unter den nordischen Ländern (OECD 2006). In Schweden waren es 2006 etwa 50\% (Skolverket 2009). In Finnland ist die gesetzliche Vorgabe bezüglich akademisch ausgebildeten Personals, dass jede dritte Beschäftigte in einer Tageseinrichtung entweder einen Bachelor- oder einen Master-Abschluss haben soll. Rund 30\% der Beschäftigten haben diesen Hochschulabschluss (Karila 2008). In Deutschland dagegen hatten 2008 nur 3\% der Gruppenleiterinnen und 21,6\% der freigestellten Einrichtungsleiterinnen einen akademischen Abschluss (Fuchs-Rechlin 2009). Auch wenn dieser Anteil allmählich steigt, ist ein akademischer Abschluss immer noch keine verbindliche Voraussetzung für die Gruppenleitung (oder Einrichtungsleitung) in frühpädagogischen Tageseinrichtungen.

Die offiziellen Qualifikationsanforderungen für die Ausübung des Berufs in Deutschland unterscheiden sich von den nordischen Ländern nicht nur in den Mindestvoraussetzungen, sondern auch von den Vorgaben der überwiegenden Mehrzahl der EU-Länder. In 22 der 27 EU-Länder ist die Voraussetzung für eine Stelle als Gruppenleitung eine mindestens dreijährige (früh)pädagogisch orientierte Hochschulausbildung - heute mit Bachelor-Abschluss (Oberhuemer u. Schreyer, im Druck). Allerdings - und dies ist bei jedem Versuch eines Vergleichs wichtig - gilt dies in den meisten Ländern nur für die Arbeit in Vorschuleinrichtungen für 4- bis 6-Jährige bzw. 3- bis 6-Jährige. Lediglich in den nordischen und baltischen Ländern (und in Slowenien) gilt diese Regelung für die Bildungs- und Erziehungsarbeit mit allen Altersgruppen ( 0 bis 6/7 Jahre) vor der Einschulung. In der Mehrheit der EU-Länder haben ministeriell getrennt gesteuerte Kita-Systeme zu unterschiedlichen Standards bezüglich der erforderlichen Personalqualifikation für die Arbeit mit Kindern unter bzw. über 3 Jahren geführt. Aber auch in diesen Ländern werden die ersten Hochschulstudiengänge speziell für die Arbeit mit unter 3-Jährigen entwickelt und demnächst eingeführt, so zum Beispiel in Ungarn und Rumänien (Oberhuemer u. Schreyer, im Druck).

Mit Blick auf den Bologna-Prozess bezüglich eines einheitlichen europäischen Hochschulrahmens bis 2010 ist festzustellen, dass sich auch die Ausbildungen im frühpädagogischen Bereich im Umbruch befinden. Im nächsten Abschnitt werden aktuelle Entwicklungstendenzen bezüglich der Ausbildungskonzepte und -strukturen in Dänemark, Finnland und Schweden herausgearbeitet. 


\section{Beständigkeit und Wandel der Ausbildungen in Dänemark, Finnland und Schweden}

\subsection{Dänemark}

In Dänemark ist die Berufsbezeichnung für die Kernfachkräfte in Tageseinrichtungen pcedagog (wörtlich: Pädagoge). Pcedagoger absolvieren eine 31/2jährige Ausbildung an einer berufsqualifizierenden Hochschule (Professionshøjskole). Dieser Ausbildungsgang qualifiziert sie nicht nur für die Arbeit in frühpädagogischen Einrichtungen, sondern auch für die Bildungs- und Erziehungsarbeit in Vorschulklassen an Grundschulen, in Schulklubs und Freizeiteinrichtungen für schulaltrige Kinder, in Kinder- und Jugendheimen sowie in diversen nicht-formellen Settings für Jugendliche und auch Erwachsene mit besonderem Förder- und Unterstützungsbedarf. Der Studiengang besteht bereits seit 1992. Damals wurden drei bisher getrennte Ausbildungsgänge mit den Schwerpunkten Kindergartenpädagogik, Freizeitpädagogik und Förderpädagogik in einer Breitbandausbildung zusammengeführt. Heute heißt der berufliche Abschluss: Bachelor in Social Education.

2004, zwölf Jahre nach der Einführung der Ausbildung zum poedagog, legte die Regierung eine Reihe von Reformempfehlungen vor, die auf der Grundlage einer nationalen Evaluation der Ausbildung durch das Dänische Evaluationsinstitut (http://www.eva.dk) erfolgten. Während diese Empfehlungen keine grundsätzliche Kritik der radikalen Form der Breitbandausbildung enthielten, wurden die Ausbildungsstätten aufgefordert, (1) eine Spezialisierungsphase mit einer spezifischen Nutzergruppe anzubieten, (2) die Wissensbasis der Studierenden durch die zusätzlichen Fachrichtungen Pädagogische Soziologie und Pädagogische Anthropologie zu erweitern und (3) die 16-monatigen Praktika zu kürzen.

Auf dieser Grundlage folgte 2007 ein verbindliches Rahmencurriculum für die Ausbildung (Jensen 2009). Die Länge der Ausbildung bleibt wie bisher bei 210 ECTS-Punkten. Die Praktika sind um nur einen Monat gekürzt worden und finden zu drei Zeitpunkten im Laufe der 31/2 Jahre statt. Statt einzelner Fächer werden nun breite Lernfelder definiert: Theorie und Praxis der Erziehungswissenschaft; dänische Kultur und Kommunikation; Individuum, Institution und Gesellschaft. Aus den folgenden drei curricularen Bereichen wählen die Studenten jetzt nur einen Schwerpunkt: a) Gesundheit, Körper und Körperübungen; b) Ausdruck, Musik und Theater; c) Kunst und Werken, Naturwissenschaft und Technologie, was eine Einschränkung des bisherigen breiten Kompetenzspektrums in den musischen Fächern impliziert. Eine weitere für Dänemark signifikante Änderung ist die Einführung einer deutlicheren Arbeitsfeldspezialisierung. Wenn Studierende ein besonderes Interesse in der frühkindlichen Bildung- und Erziehungsarbeit zeigen, verbringen sie nun mindestens sechs Monate ihrer 15-monatigen Praktikumszeit in einer frühpädagogischen Einrichtung. Während der Praktikumszeit werden sie bezahlt (außer in den ersten drei Monaten) und währenddessen als Teil des offiziellen Anstellungsschlüssels mitgezählt, was ein kontroverser Punkt für die professionsbezogene Gewerkschaft BUPL ist, in der nahezu alle poedagoger Mitglieder sind.

Es gab aber nicht nur inhaltliche Neuorientierungen. 2008 fand auch ein radikaler Umbau der Hochschullandschaft in Dänemark statt. Während poeedagoger bisher an 32 berufsspezifischen Hochschulseminarien ausgebildet wurden, sind diese Seminarien 
zusammen mit rund 70 anderen Hochschuleinrichtungen für die Lehrer- und Sozialarbeiterausbildung nun Teil von insgesamt acht Gesamthochschulen (university colleges). Selbstverständlich ist diese Umstrukturierung nicht ohne Auswirkung auf die Administration und auch auf die konkrete Gestaltung der Studiengänge geblieben.

Besonders interessant in Dänemark ist das inklusive Modell der flexiblen Zugangsvoraussetzungen (Jensen 2009). Kandidaten werden nach einem Quotensystem zugelassen. Quote 1 (=35\% der Aufnahmen) rekrutiert die Kandidaten mit den besten Schulnoten. Quote 2 (=65\% der Aufnahmen) besteht aus Kandidaten, die nach ihren jeweils erworbenen Kompetenzen und Qualifikationen eingestuft werden. Diese berücksichtigen zum Beispiel einen bereits erworbenen beruflichen Abschluss, feldrelevante Berufserfahrung oder einen Aufenthalt im Ausland.

\subsection{Finnland}

Die Ausbildung als lastentarhanopettaja (wörtlich: Kindergarten-Lehrerin) wurde 1995 als akademischer Studiengang an der Universität angesiedelt und die bisherigen Ausbildungseinrichtungen für diese Berufsgruppe wurden aufgelöst. Dieses waren keine radikale Veränderungen, weil bereits seit 1973 die Ausbildungsstätten zum Teil an den Universitäten angegliedert waren und es bestanden ohnehin enge Kooperationsformen (Oberhuemer u. Ulich 1997). Nach der dreijährigen Ausbildung mit einem erziehungswissenschaftlichen Bachelor-Abschluss (180 ECTS-Punkte) können die Absolventen als Gruppen- und Einrichtungsleiterinnen in Tageseinrichtungen für 0- bis 6-Jährige und auch in den Vorschulklassen für 6- bis 7-Jährige arbeiten. Während ein Masters-Abschluss die bevorzugte Qualifikation für Leitungskräfte ist, ist dies keine verbindliche Vorgabe.

Die Struktur der Ausbildung variiert je nach Universität, wie auch die Gewichtung der Frühpädagogik innerhalb des Studiums. An der Universität Helsinki gliedert sich zum Beispiel das Ausbildungscurriculum für angehende Kindergarten-Lehrkräfte in vier Schwerpunkte (Onnismaa 2009) (vgl. Übersicht 1).

Allerdings sind die lastentarhanopettaja nicht die einzigen Fachkräfte, die in Kindertageseinrichtungen arbeiten. Im finnischen Gesetz 2005 zur Qualifikation von Angestellten in der sozialen Wohlfahrt wird festgelegt, dass nur jede dritte Beschäftigte einen Bachelor/Master der Erziehungswissenschaft oder Sozialwissenschaft vorweisen muss; die beiden anderen Fachkräfte sollen entweder eine post-sekundäre oder eine Sekundarstufen-II-Qualifikation im Sozial- und Gesundheitswesen haben. Diese Fachkräfte sind in der Regel Personen mit einer Ausbildung als Kinderbetreuerin (lastenhoitaja) oder Erstbetreuerin (lähihoitaja) - meistens mit einer einjährigen Spezialisierung auf frühkindliche Erziehung und Betreuung. Eine zusätzlich qualifizierte Kindergarten-Lehrkraft unterstützt Kinder mit Behinderungen.

Früher musste jede dritte Fachkraft das pädagogische Studium als Kindergarten-Lehrerin (lastentarhanopettaja) absolvieren, heute kann diese dritte Person auch eine Sozialerzieherin (sosionomi, $A M K$ ) mit einem sozialwissenschaftlichen Bachelor-Abschluss einer polytechnischen Hochschule sein. Dabei handelt es sich um eine breit angelegte sozialpädagogisch-sozialarbeitsorientierte Ausbildung mit einer Spezialisierung von wenigstens 60 ECTS in der frühen Kindheit. In finnischen Tageseinrichtungen findet man also multiprofessionelle Teams - und der Begriff der Multiprofessionalität taucht immer wieder 
Übersicht 1: Struktur des Ausbildungscurriculums zur Kita-Fachkraft, Universität Helsinki, 2008/09 (Die Zahlen in Klammern beziehen sich auf ECTS-Punkte)

1 Sprache und Kommunikation 20

u. a. Wissenschaftliches Schreiben und sprachliche Kommunikation (5);

Fremdsprache (3)

2 Grundstudium Erziehungswissenschaft

u. a. Sozio-kulturelle Theorien (8); Psychologische Grundlagen der frühkindlichen Bildung/Erziehung (8); Pädagogische Grundlagen (6); Forschungsphilosophie und Forschungsmethoden (3)

3 Aufbaustudium Erziehungswissenschaft

u. a. Frühkindliche Bildung/Erziehung (22); Forschungsmethoden und

Seminararbeiten (10); Bachelorarbeit (6)

4 Curriculare Grundlagen für die Arbeit in Tageseinrichtungen und

Vorschulklassen u. a. Bildungsbereiche (15); ästhetische Bildung (3);

Praktika (15); Wahlfächer (34)

Gesamt

180 ECTS

Quelle: Onnismaa 2009

in den offiziellen Dokumenten auf. Es kann aber vorkommen, je nach den Anstellungsbedingungen der einzelnen Kommunen, dass es in einer Kindertageseinrichtung keine pädagogische ausgebildeten Kindergarten-Lehrkräfte gibt, weil die Kommune stattdessen eine Sozialerzieherin (sosionomi) eingestellt hat (Karila 2008, S. 216). Außerdem sind die Aufgabenverteilungen und Verantwortlichkeiten in den Teams nicht - wie früher - professionsspezifisch geklärt. So gestaltet sich die multiprofessionelle Zusammenarbeit nicht ohne Spannungen und Territoriumskämpfe (Kinos 2008).

\subsection{Schweden}

Seit 1977 werden Kita-Fachkräfte in Schweden an Universitäten ausgebildet (Grahn 2008, S. 84). Damit hat Schweden die längste Tradition einer akademischen Ausbildung unter den nordischen Ländern. Während diese Fachkräfte bis 2001 eine dreijährige Ausbildung speziell für den Bereich der Pädagogik der frühen Kindheit absolviert haben, sind sie heute in eine einheitliche Lehrer-Ausbildung eingegliedert. Im Rahmen der allgemeinen bildungspolitischen Reforminitiativen nach 1996 und der Zuordnung der Kitas zum Bildungsbereich wurde auch die Anschlussfähigkeit zwischen Tageseinrichtungen und Schulen konsequent angestrebt: durch die Formulierung institutionsübergreifender curricularer Grundsätze und Ziele; durch die strukturell gesicherte Zusammenarbeit von Kita-Fachkräften, Freizeit- und Grundschulpädagogen in den Vorschulklassen an Grundschulen; und nicht zuletzt - seit 2001 - durch eine integrierte Ausbildung für die pädagogische Arbeit mit Kindern im Alter von 1 bis 18 Jahren. Kita-Fachkräfte werden jetzt als Lärare (wörtlich: Lehrer) ausgebildet: für die Arbeit in Tageseinrichtungen für 1- bis 5-Jährige, in Vorschulklassen an Grundschulen für 6-Jährige und auch für die Zusammenarbeit mit Grundschullehrkräften und Freizeitpädagogen in den ersten 4 Schuljahren (mit 7- bis 11-Jährigen). Bestimmte Teile der Ausbildung (z.B. erziehungswissenschaftliche Grundlagen) finden auch zusammen mit den angehenden Lehrern der Sekundarstufe I 
statt. Die Ausbildung dauert wie in Dänemark 31/2 Jahre. Diejenigen, die mit jungen Kindern arbeiten wollen, müssen mindestens 140 ECTS-Punkte akkumulieren (pro Semester 20 Kreditpunkte). Eine Spezialisierung in Frühpädagogik findet statt, Studenten entscheiden sich aber erst nach etwa anderthalb Jahren für ein bestimmtes Arbeitsfeld.

Das Ausbildungscurriculum gliedert sich in drei Schwerpunkte (Karlsson Lohmander 2007, S. 1268):

(1) Grundstudium - z.B. erziehungswissenschaftliche Grundlagen, Förderpädagogik, Entwicklung von Kindern sowie Querschnittsthemen wie zum Beispiel Grundsätze und Wertorientierungen in der Bildungs- und Erziehungsarbeit, das Demokratieprinzip, Gender-Fragen (60 ECTS-Punkte, mindestens 10 davon in Praxiseinrichtungen);

(2) Fächerbezogene Studien - Sprache, Literacy, Mathematik, Umweltbildung usw. (40 ECTS-Punkte, mindestens 10 davon in Praxiseinrichtungen);

(3) Spezialisierung - Vertiefung bereits erworbener Kenntnisse in einem bestimmten Bereich, z. B. Soziologie der Kindheit, Erwachsenenbildung, internationale Perspektiven (20 ECTS-Punkte).

In diesem Rahmen entwickelt jede Universität einen eigenen Studienplan.

Die erste nationale Evaluation der neuen Lehrerbildung fand 2005 statt (Karlsson Lohmander 2007, S. 1269). Bereits in diesem frühen Stadium wurden einige Probleme deutlich. Bezüglich der Frühpädagogik tendieren Studierende dazu, eher das Arbeitsfeld Grundschule $\mathrm{zu}$ wählen. Vermutlich wirken sich die noch bestehenden Unterschiede in Vergütung und Arbeitsbedingungen in den zwei Berufsfeldern negativ auf eine Entscheidung für die Arbeit mit jüngeren Kindern aus. Es hat sich auch gezeigt, dass es den Studierenden im neuen System schwerer fällt, eine professionelle Identität zu entwickeln. In der bisherigen Ausbildung zur Vorschulpädagogin, die stark in Praxisbezügen verortet war, war es eher möglich, ein klares professionelles Selbstverständnis zu entwickeln.

Aufgrund von Problemen in der Personalrekrutierung im Bildungssystem legte im Dezember 2008 eine Regierungskommission einen neuen Vorschlag für die Lehrer-Ausbildung vor, der sich noch in der Konsultationsphase befindet (Karlsson Lohmander 2009). Nach diesem Vorschlag soll die Ausbildung nicht mehr einheitlich, sondern in vier klar abgegrenzte Berufsausrichtungen gegliedert werden: (1) Frühpädagogische Tageseinrichtung (förskola) für 1- bis 5-Jährige; (2) Vorschulklasse und die ersten 3 Schuljahre für 6- bis 9-Jährige; (3) Schuljahre 4 bis 6 für 10- bis 12-Jährige; (4) Freizeitzentren für die außerschulische Arbeit mit Schulkindern. Die Ausbildung soll auf 3 Jahre gekürzt werden. Kita-Fachkräfte werden nur mit einer einjährigen Zusatzausbildung in Vorschulklassen an Grundschulen arbeiten können. Falls sich das schwedische Parlament für dieses Modell entscheidet (im Feld gibt es viel Opposition dazu), wird die neue Lehrerbildung im Herbst 2010 eingeführt, also nur 9 Jahre nach der Einführung der aktuellen Studienordnung.

\section{Professionsprofile frühpädagogischer Kernfachkräfte - ungeklärtes Terrain}

Diese Beispiele zeigen, wie unterschiedlich noch die Visionen, Konzepte und Strukturen der Ausbildungen für die Bildungs- und Betreuungsarbeit in frühpädagogischen Tages- 
einrichtungen sind - sogar in drei benachbarten nordischen Ländern. Während Einigkeit über die formale Qualifikationsanforderung für diesen Beruf besteht - sie soll ein Hochschulabschluss sein -, gehen die Vorstellungen über das Profil für die Profession deutlich auseinander.

In Dänemark ist das Berufsprofil arbeitsfeldübergreifend konzipiert und außerhalb des Bildungssystems verortet. Es ist das Profil einer sozialpädagogischen Fachkraft mit unterschiedlichem Professionsverständnis und gegenüber der Schullehrkraft abgegrenztem Tätigkeitsfeld. Die Ausbildung qualifiziert für die Arbeit mit Menschen über die Lebensspanne, auch wenn etwa Dreiviertel der Absolventen nach dem Studium einer Arbeit mit Kindern von 0 bis 10 Jahren nachgeht (Jensen 2009). Das zugrundeliegende Verständnis von Pädagogik und Menschenbildung ist interdisziplinär - mit sozialen, kulturellen und gesundheitsorientierten Bezügen. Kenntnisse über die frühe Kindheit werden in eine weite gesellschaftliche Perspektive eingebettet.

In Finnland - wenn wir hier die pädagogisch ausgebildeten Kindergarten-Lehrkräfte in den Blick nehmen - ist das Berufsprofil ein ganz anderes. Die Kindergarten-Lehrerin ist eine Spezialistin in Sachen frühkindlicher Bildung und Erziehung für Kinder im Alter von 0 bis 7 Jahren. Die Arbeitsfelder sind klar begrenzt: Tageseinrichtungen und Vorschulklassen. Die Ausbildung ist pädagogisch ausgerichtet, dies aber in einem sozialwohlfahrtlichen policy-Kontext. Während die paedagoger in Dänemark von einer meist unausgebildeten Assistentin unterstützt werden, müssen die Kindergarten-Fachkräfte in Finnland ihre Position in einem multiprofessionellen Team immer wieder neu behaupten.

In Schweden ist das Berufsprofil wiederum anders: institutionsübergreifend, jedoch innerhalb eines breit definierten Bildungssystems, das auch Kindertageseinrichtungen und außerschulische Angebote für Schulkinder einbezieht. Die Kita-Fachkräfte werden als ,Lehrer" bezeichnet, sie sind in das schulische Bildungssystem eingegliedert und können dort in den ersten vier Jahrgangsklassen mitarbeiten, in einem Schulsystem, das auf den gleichen Grundprinzipien und Wertorientierungen wie der Kita-Bereich fußt. Ist dies das modernere Berufsprofil? Auch wenn manche diese Frage mit ,ja' beantworten mögen, zeigen die neuesten Entwicklungen in Schweden, wie fragil das sich neu entwickelnde Profil vermutlich ist, wie stark dagegen die etablierte Position der Schule gegenüber der förskola - auch wenn sie ,neu gedacht“ wird.

\section{Nordische Beispiele als Inspiration für den Klärungsprozess in Deutschland?}

Die hier dargestellten Unterschiede und Ambivalenzen spiegeln die in vielen Ländern vorhandenen ungeklärten Zuständigkeiten und Visionen für den frühpädagogischen Sektor wider: Bildung oder Soziales? - Oder ein soziokulturelles Verständnis von Bildung? Die Beantwortung dieser Fragen stellt sich in Deutschland wohl noch komplexer dar als in den nordischen Ländern. In einer Analyse der aktuellen Bestrebungen zur Akademisierung der Erzieherinnen-Ausbildung in Deutschland wird die noch bevorstehende Professionalisierungsaufgabe als eine „Mehr-Ebenen-Herausforderung“ beschrieben (König u. Pasternack 2008, S. 146). Neben den spezifisch gemeinten Herausforderungen für die Ausbildungsstrukturen und -konzepte ist hiermit auch generell die Komplexi- 
tät des Systems der frühpädagogischen Tageseinrichtungen für Kinder in Deutschland gemeint: ein föderales Modell der Verantwortung mit geringer Entscheidungsbefugnis auf Bundesebene und großer Gestaltungskraft auf kommunaler Ebene; eine bunte Vielfalt von öffentlichen und nicht-öffentlichen Kita-Trägern und Interessengruppen. Auch die Einrichtungsprofile - und damit auch die Anforderungsprofile an die Ausbildung - differenzieren sich immer weiter: neben den klassischen Kindergärten und Kinderkrippen und altersübergreifenden Tageseinrichtungen entstehen politisch gesteuerte Alternativmodelle mit ganz unterschiedlichen Konzepten wie die Kinder- und Familienzentren oder die Bildungshäuser für Kinder von 3 bis 10 Jahren.

In der allgemeinen sozialpädagogischen und soziokulturellen Ausrichtung des dänischen Professionsprofils ist eine Ähnlichkeit zum traditionellen deutschen Berufsprofil der Erzieherin festzustellen, das sich aus der in Deutschland historisch gewachsenen Zuordnung des Kindergartens zum Sozialwesen entwickelt hat. Das finnische Modell der frühpädagogischen Fachkraft (,Kindergarten-Lehrkraft“), die auch den Übergang in die Schule gestaltet, zeigt einen Weg der Spezialisierung, den es traditionell hier in Deutschland nicht gab und der mit Blick auf die Umsetzung der neuen Bildungspläne immer wieder gefordert wird. Schweden bietet ein Modell, das anstrebt, Kita und Schule konzeptionell im Kontext eines breiten Bildungsverständnisses zu verbinden. In Schweden muss aber ein klares Berufsprofil zu allererst geschaffen werden - falls die Ausbildungsreform nicht rückgängig gemacht wird, denn Ausbildungsentwicklungen ohne Bezug zu den Erfordernissen des Berufsfeldes haben sicherlich keine Zukunft (vgl. Rauschenbach 2006; Rauschenbach u. Schilling 2009).

Die uralte Grundsatzfrage nach notwendiger Spezialisierung bei gleichzeitig erforderlicher Generalisierung in der Ausbildung muss wohl immer wieder neu gestellt werden. Hier können die drei nordischen Beispiele zum Klärungsprozess in der noch nicht abgeschlossenen Diskussion um das frühpädagogische Professionsprofil in Deutschland beitragen - nicht als Modell, nicht als Mythos, sondern als handfeste Argumentationshilfe.

Open Access: Dieser Artikel unterliegt den Bedingungen der Creative Commons Attribution Noncommercial License. Dadurch sind die nichtkommerzielle Nutzung, Verteilung und Reproduktion erlaubt, sofern der/die Originalautor/en und die Quelle angegeben sind.

\section{Literatur}

Allodi, M. W. (2007). Child care and pre-schools in Sweden: An overview of practice, tendencies and research. Richerche di Pedagogia e Didattica - Pedagogia e Didattica dei Servizi Educativi, 2, 1-10.

Autorengruppe Bildungsberichterstattung. (2008). Bildung in Deutschland 2008. Ein indikatorengestützter Bericht mit einer Analyse zu Übergängen im Anschluss an den Sekundarbereich I. Bielefeld: W. Bertelsmann.

BMBF - Bundesministerium für Bildung und Forschung. (2007). Education unites - Conference of the European Education Ministers, 1-2 March 2007. Final Communiqué. http://www.bmbf. de/pub/kommunique_eu_heidelberg_d.pdf. Zugegriffen 27. März 2009. 
Bundeszentrale für politische Bildung. (2008). Die soziale Situation in Deutschland. http://www. bpb.de/wissen/37OUAU,0,0,Die_soziale_Situation_in_Deutschland.html. Zugegriffen 23. März 2009.

Deutsches Jugendinstitut. (2008). Zahlenspiegel 2007. Kindertagesbetreuung im Spiegel der Statistik.http://www.bmfsfj.de/bmfsfj/generator/Publikationen/zahlenspiegel2007/7-Das-personal-in-kindertageseinrichtungen-entwicklungen-und-herausforderungen0.html. Zugegriffen 20. März 2009.

Ebert, S. (2006). Erzieherin - ein Beruf im Spannungsfeld von Gesellschaft und Politik. Freiburg: Herder.

Eurostat. (2005). Employment rates lower and part-time rates higher for women with children (News Release, Nr. 49.). http://europa.eu/rapid/pressReleasesAction.do?reference=STAT/05/ 49\& format=HTML\&aged=0\&language=EN\&guiLanguage=en. Zugegriffen 15. Mai 2009.

Eurostat. (2008). Childcare in the EU in 2006. http://europa.eu/rapid/pressReleasesAction.do?reference=STAT/08/172\&type=HTML. Zugegriffen 19. Mai 2009.

Forschungsverbund DJI/TU Dortmund. (2008). Kindertagesbetreuung in Deutschland. Kennzahlen - Indikatoren - Daten. http://www.akjstat.uni-dortmund.de/akj/Downloads/Kita2008.pdf. Zugegriffen 30. März 2009.

Fuchs-Rechlin, K. (2009). Akademisierung in Kindertageseinrichtungen - Schein oder Sein? KomDat Jugendhilfe, 12(1), 18-19.

Grahn, M. (2008). Das Lehramtsstudium an der Universität Linköping, Schweden. In U. Carle \& B. Daiber (Hrsg.), Das Kind im Blick. Eine gemeinsame Ausbildung für den Elementarbereich und die Grundschule. Entwicklungslinien und Forschungsbefunde (S. 84-90). Baltmannsweiler: Schneider Hohengehren.

Haas, L., Chronholm, A., \& Hwang, P. (2008). Sweden. In P. Moss \& M. Korintus (Hrsg.), International Review of Leave Policies and Related Research 2008 (S. 333-345). London: Department for Business Enterprise \& Regulatory Reform. http://www.berr.gov.uk/files/file47247. pdf. Zugegriffen 9. Okt. 2009.

Hännikäinen, M. (2009). Bildung, Erziehung und Betreuung von jungen Kindern in Finnland. In M. Textor (Hrsg.), Kindergartenpädagogik. Online-Handbuch. http://www.kindergartenpaedagogik.de/1998.html. Zugegriffen 9. Okt. 2009.

Jensen, J. J. (2009). Expertenbeiträge im Auftrag des Staatsinstituts für Frühpädagogik (SEEPROProjekt). Bisher unveröffentlichtes Manuskript. Aarhus.

Karila, K. (2008). A finnish viewpoint on professionalism in early childhood education. European Early Childhood Education Research Journal, 16(2), 210-223.

Karlsson Lohmander, M. (2007). Early childhood teacher education in Sweden. In R. New \& M. Cochran (Hrsg.), Early childhood education. An international encyclopedia, Vol. 4 (S. 1266 1270). Westport: Praeger.

Karlsson Lohmander, M. (2009). Expertenbeiträge im Auftrag des Staatsinstituts für Frühpädagogik (SEEPRO-Projekt). Bisher unveröffentlichtes Manuskript. Göteborg.

Kinos, J. (2008). Professionalism - a breeding ground for struggle. The example of the finnish daycare centre. European Early Childhood Education Research Journal, 16(2), 224-241.

König, K., \& Pasternack, P. (2008). elementar + professionell. Die Akademisierung der elementarpädagogischen Ausbildung in Deutschland. Mit einer Fallstudie: Der Studiengang ,Erziehung und Bildung im Kindesalter " an der Alice Salomon Hochschule Berlin. (HoF-Arbeitsbericht 5 '08). Wittenberg: Martin-Luther-Universität Halle-Wittenberg.

Leu, H.-R., \& Schelle, R. (2009). Between education and care? Critical reflections on early childhood policies in Germany. Early Years - An International Journal of Research and Development, 29(1), 5-18. 
Oberhuemer, P. (2000). Lernkulturen - Berufskulturen: Entwicklungstendenzen in europäischen Kindertageseinrichtungen. In H. Colberg-Schrader \& P. Oberhuemer (Hrsg.), Qualifizieren für Europa. Praxiskulturen, Ausbildungskonzepte, Initiativen (S. 28-45). Baltmannsweiler: Schneider Hohengehren.

Oberhuemer, P., \& Schreyer, I. (im Druck). Kita-Fachpersonal in Europa: Ausbildungen und Professionsprofile. Opladen u. Farmington Hills, MI: Barbara Budrich.

Oberhuemer, P., \& Ulich, M. (1997). Working with young children in Europe. London: Paul Chap$\mathrm{man} /$ Sage.

Oberhuemer, P., \& Schreyer, I. unter Mitwirkung von M. J. Neuman. (2009). Fachpersonal in Kindertageseinrichtungen der Europäischen Union. Ergebnisse aus dem SEEPRO-Projekt (Systeme der Elementarerziehung und Professionalisierung in Europa). http://www.bmfsfj.bund. de/bmfsfj/generator/BMFSFJ/Kinder-und-Jugend/kinderbetreuung.html. Zugegriffen 9. Okt. 2009.

OECD - Organisation for Economic Co-operation and Development. (2006). Starting strong II. Early childhood education and care. Paris: OECD.

OECD - Organisation for Economic Co-operation and Development. (2007). Babies and bosses. Reconciling work and family life. Paris: OECD.

Onnismaa, E.-L. (2009). Expertenbeiträge im Auftrag des Staatsinstituts für Frühpädagogik (SEEPRO-Projekt). Bisher unveröffentlichtes Manuskript. Helsinki.

Pestalozzi-Fröbel-Verband. (1977). Zur Erzieherausbildung in der Bundesrepublik Deutschland. Tatsachen, Überlegungen, Forderungen. Berlin: Eigenverlag.

Rauschenbach, T. (2006). Ende oder Wende? Pädagogisch-soziale Ausbildungen im Umbruch. In A. Diller \& T. Rauschenbach (Hrsg.) (2006), Reform oder Ende der Erzieherinnenausbildung? Beiträge zu einer kontroversen Fachdebatte (S. 13-34). München: Verlag Deutsches Jugendinstitut.

Rauschenbach, T., \& Schilling, M. (2009). Demografie und frühe Kindheit. Prognosen zum Platzund Personalbedarf in der Kindertagesbetreuung. Zeitschrift für Pädagogik, 55, 17-36.

Robert Bosch Stiftung. (Hrsg.) (2008). Frühpädagogik studieren - ein Orientierungsrahmen für Hochschulen. Stuttgart: Eigenverlag.

Schrader, H. (1973). Professionalisierung der Erzieher im Rahmen vorschulischer Curriculumentwicklung. In J. Zimmer (Hrsg.), Curriculumentwicklung im Vorschulbereich (Bd. 1, S. 285-318). München: Piper.

Siraj-Blatchford, I. (2004). Quality teaching in the early years. In A. Anning, J. Cullen, \& M. Fleer (Hrsg.), Early childhood education. Society and culture (S. 137-148). London: Sage.

Skolverket - Swedish National Agency for Education. (2009). Pre-school staff - national level. http://www.skolverket.se/sb/d/1613/a/14761\#paragraphAnchor3. Zugegriffen 30 März 2009.

Statistics Denmark. (2009). Population and elections. http://www.statbank.dk/statbank5a/default. asp?w=1024. Zugegriffen 26. Mai 2009.

WiFF - Weiterbildungsinitiative Frühpädagogische Fachkräfte. (2009). Die Datenlage. http://www. weiterbildungsinitiative.de/die-datenlage/fruehpaedagogische-ausbildungslandschaft/studiengaenge-nach-abschluss.html. Zugegriffen 29. März 2009. 